\title{
Computing Constrained Minimum-Width Annuli of Point Sets*
}

\author{
Mark de Berg ${ }^{\dagger} \quad$ Prosenjit Bose ${ }^{\ddagger} \quad$ David Bremner ${ }^{\S}$ \\ Suneeta Ramaswami" Gordon Wilfongl|
}

November 29, 1996

\begin{abstract}
We study the problem of determining whether a manufactured disc of certain radius $r$ is within tolerance. More precisely, we present algorithms that, given a set of $n$ probe points on the surface of the manufactured object, compute the thinnest annulus whose outer (or inner, or median) radius is $r$ and that contains all the probe points. Our algorithms run in $O(n \log n)$ time.
\end{abstract}

\section{Introduction}

One of the fundamental aspects of the manufacturing sciences is quality control for various objects that are built by some physical process, i.e. the measurement of the "shape" and "size" of such manufactured objects in order to determine whether they conform sufficiently to the specified ideal measures. Metrology is the science of measurement. One of its subareas is tolerancing (or dimensional tolerancing), which addresses questions pertaining to specifications of the tolerance of shape characteristics and the methodology used to measure it. Coordinate Measurement Machines

\footnotetext{
${ }^{*}$ This research was partially supported by the Dutch Organization for Scientific Research N.W.O, NSERC Canada and FCAR Quebec. We also thank the Bellairs Research Institute in Barbados for the use of their facilities.

${ }^{\dagger}$ Department of Computer Science, Utrecht University, PO Box 80.089, 3508 TB Utrecht, The Netherlands

‡Département de Mathematique et Informatique, Université du Québec à Trois-Rivières, TroisRivières, Québec, Canada

${ }^{\S}$ School of Computer Science, McGill University, Montréal, Québec, Canada

"School of Computer Science, McGill University, Montréal, Québec, Canada

"Bell Laboratories, Lucent Technologies, Murray Hill, New Jersey, USA
} 


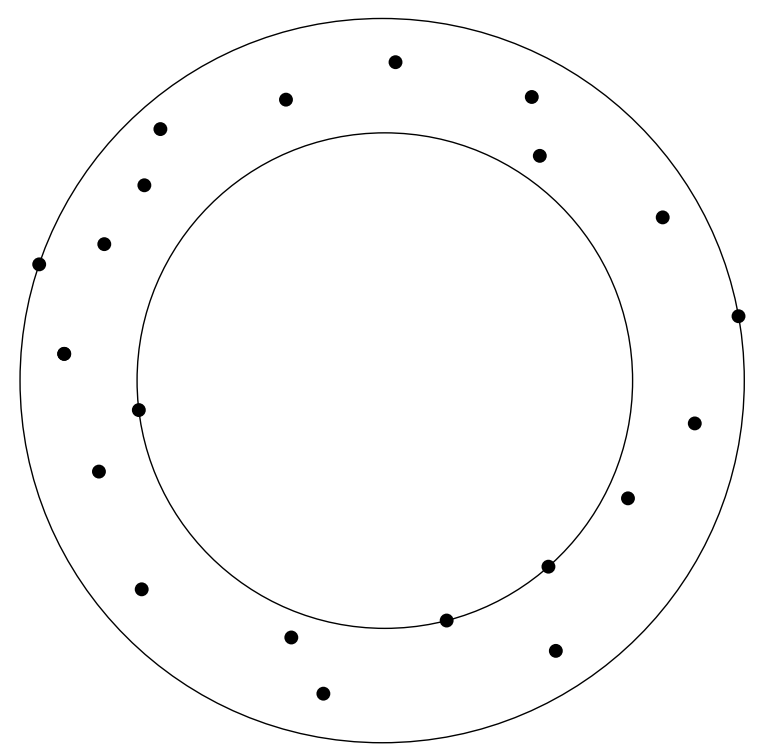

Figure 1: A feasible annulus for a set of points.

(CMMs) are machines that take measurements on a physical object by probing it and record such information (for example, this could be the coordinate values of various points on the object). This information may then be used to compute various characteristics of the object in order to determine whether they lie within the specified tolerance. Such problems fall in the domain of computational metrology. Many of these problems have a rich geometric flavor and the advent of CMMs has led to much interaction between computational geometry and metrology.

A fundamental and well-studied problem in tolerancing metrology is the problem of measuring the roundness of an object. In this case, the manufactured objects are round (circular) and the CMM gives the coordinate values of $n$ points sampled on the boundary of the manufactured object. The goal is to determine whether the object is close to circular or not. This is the problem of computing the minimum width annulus containing the given set of points. See Figure 1 for an example of an annulus of a set of points. If the thickness of such an annulus is less than the specified tolerance, the manufactured object is acceptable. This is, in fact, the measure used by the American National Standards Institute (see Foster [9], pp. 40-42) and the International Standards Office for testing roundness.

\subsection{Previous Work}

The minimum width annulus problem has been well-studied. Rivlin [18] first showed that the minimum width annulus of $n$ points is either the width of the set (the width of a set of points is the thinnest strip that contains all the points) or must have two 


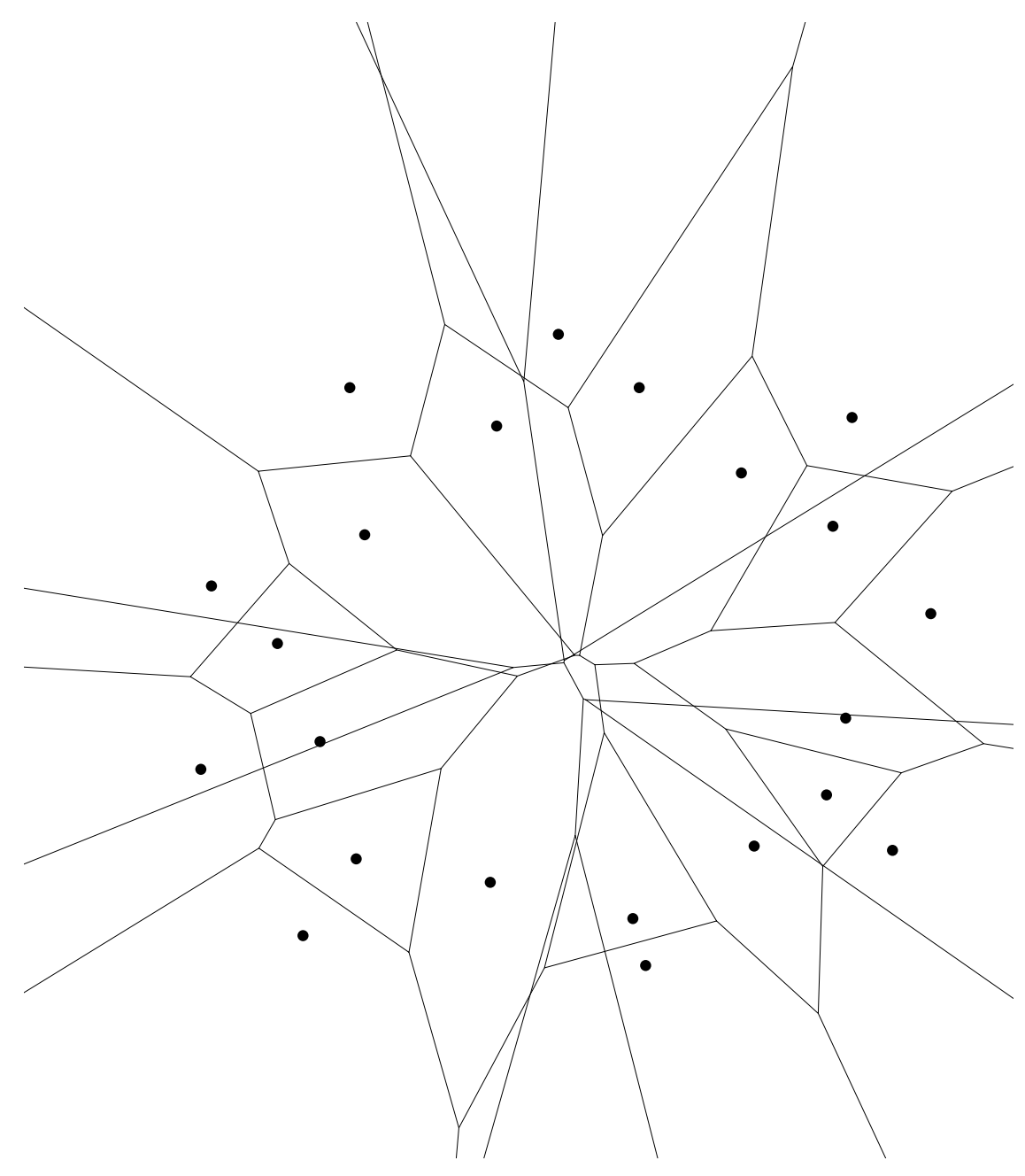

Figure 2: The union of the nearest and furthest point Voronoi diagrams of a set of points.

points on the inner circle and two points on the outer circle of the annulus. In the case of the former, the radius of the annulus is infinite and in the case of the latter, it is finite. This result implies that if the radius of the minimum width annulus of a set $S$ of $n$ points is finite, then its center must lie on an intersection point of the furthest-point Voronoi diagram of $S$ and nearest-point Voronoi diagram of $S$; see Figure 2. Smid and Janardan [22] give an alternate proof of Rivlin's characterization of the minimum width annulus of a set of planar points.

An algorithm for this problem was given by Ebara et al. [7] in 1989, where they give an $O\left(n^{2}\right)$ time algorithm for the problem by computing the union of the nearest and furthest-point Voronoi diagrams of the input set of $n$ points. This algorithm can be improved to run in $O(n \log n+k)$ time by using the planar map overlay algorithm 
due to Guibas and Seidel [11], where $k$ represents the number of intersection points between the nearest and furthest-point Voronoi diagrams. In the worst case $k$ can be $\Theta\left(n^{2}\right)$, thus this algorithm has worst case running time of $\Theta\left(n^{2}\right)$. This algorithm was re-discovered in the manufacturing community by Roy and Zhang in 1992 [19].

Recently, Bose and Devroye [5] have shown that the expected number of intersections between the furthest-point and nearest-point Voronoi diagram of a random point set is linear (the set of points is drawn from a density bounded from 0 and $\infty$ on a convex compact set). This implies that the above algorithm has $O(n \log n)$ expected running time in this case.

In the case where the points are not random, the above characterization for the center of the minimum-width annulus has also been used to obtain algorithms with improved running times, by using Megiddo's parametric search [15] and other techniques. Agarwal et al. [1, 2, 3] obtain randomized algorithms with running times that are $o\left(n^{2}\right)$. The best-known algorithm to date appears in [2] and has an expected running time of $O\left(n^{3 / 2+\epsilon}\right)$, where $\epsilon>0$ is an arbitrarily small constant.

Faster running times have been demonstrated for some special cases as well. For the case when the input is not a set of points but a simple polygon $P$ of $n$ vertices, the boundary edges of $P$ are required to be within the annulus. For this problem, Lai and Wang [13] gave an $O(n \log n+k)$ time algorithm, where $k$ is the number of intersections between the medial axis of $P$ and the furthest neighbor Voronoi diagram of $P$. An algorithm for this special case was also given in [14]. For the case when the input is a convex polygon, Swanson [23] gives a linear-time algorithm (even though the number of intersections between the medial axis and furthest neighbor Voronoi diagram of a convex polygon might still be as high as $\Omega\left(n^{2}\right)$ ). García et al. [10] show that even when the input is a set of points in convex position, the minimum-width annulus can be found in linear time.

\subsection{Our Results}

Suppose we would like to manufacture a disc of radius $r$. There are two aspects of the produced object that we can test, namely whether it is round enough and whether its size (that is, radius) is appropriate. Computing the smallest width annulus amounts to testing only the roundness of the object. In this paper, we shall test both aspects simultaneously. Thus we shall look for the smallest width annulus of radius $r$. We consider three versions of this problem in which the radius of an annulus is defined to be the radius of its outer circle, its inner circle, or its median circle (that is, the circle in between and equidistant from the outer and inner circle).

Note that the number of degrees of freedom of the annulus in our restricted settings is one less than in the general setting, where one wishes to compute the smallest width annulus without constraints on either its inner or outer radius. We show how 
to use this fact to improve upon the $O\left(n^{3 / 2+\epsilon}\right)$ bound given by Agarwal et al.[2] for the general case. Our algorithms for the three restricted settings each have only $O(n \log n)$ running time. Moreover, they are fairly simple, and should present no real problems to an implementor.

\section{Notation and Definitions}

In this section, we establish notation that will be used throughout the paper and also give the relevant geometric definitions. These definitions are standard; see O'Rourke [16] or Preparata and Shamos [17] for more details. The input set of points will always be denoted by $S=\left\{s_{1}, s_{2}, \ldots, s_{n}\right\}$. The Euclidean distance between two points $p_{1}$ and $p_{2}$ in the plane is denoted by $d\left(p_{1}, p_{2}\right)$. Given a point $p$ in the plane and a radius $r$, we denote the circle with center $p$ and radius $r$ by $C(p, r)$, the closed disc with center $p$ and radius $r$ by $D(p, r)$ and the open disc with center $p$ and radius $r$ by $\widetilde{D}(p, r)$. Given a closed region $R$, we use $\operatorname{bd}(R)$ to represent the boundary of $R$ and $\operatorname{int}(R)$ to represent the interior of $R$ i.e. the set $R-\operatorname{bd}(R)$.

The following geometric terms will be used frequently and we define them here: We say a closed, connected region in the plane is a circle-gon if it is a convex region bounded by circular arcs. Given a set of points $S$ and a closed bounded region $F$, a largest empty circle with center in $F$ is a circle, say $C(p, r), p \in F$, of largest radius such that no points of $S$ lie in $\widetilde{D}(p, r)$. A spanning disc of a set $S$ of points in the plane is a closed disc containing $S$. A minimum spanning disc of $S$ is a spanning disc of smallest radius. The diameter of $S$ is the maximum distance between any two of its points and a diametral pair of $S$ is a pair of points in $S$ whose distance is exactly the diameter of the set of points. Given two points $a$ and $b$ on a circle $A$, define $\operatorname{arc}(a, b)$ to be the $\operatorname{arc}$ from $a$ to $b$ in the counter-clockwise direction along $A$. The straight line bisector of two points $p_{1}$ and $p_{2}$ in the plane is denoted by $\operatorname{bis}\left(p_{1}, p_{2}\right)$. Voronoi diagrams, defined below, are elegant and versatile geometric structures with numerous applications and they are used extensively in our algorithms.

- The nearest point Voronoi diagram of a set of points $S$, denoted by $\operatorname{NPVD}(S)$, is defined as follows: The Voronoi region $V\left(s_{i}\right)$ associated with an element $s_{i}$ from $S$ is the set of all points in the plane that are closer (or as close) to $s_{i}$ than to any other element in $S$ and $\operatorname{NPVD}(S)$ is the union of all the Voronoi regions $V\left(s_{i}\right), s_{i} \in S$. See Figure 3 . The boundary edges between Voronoi regions are called Voronoi edges and the vertices of the diagram are called Voronoi vertices.

- The furthest point Voronoi diagram of a set of points $S$, denoted by $\operatorname{FPVD}(S)$, is defined similarly, except that now the Voronoi region associated with an element $s_{i}$ from $S$ is the set of all points in the plane that are farther (or as far) from 


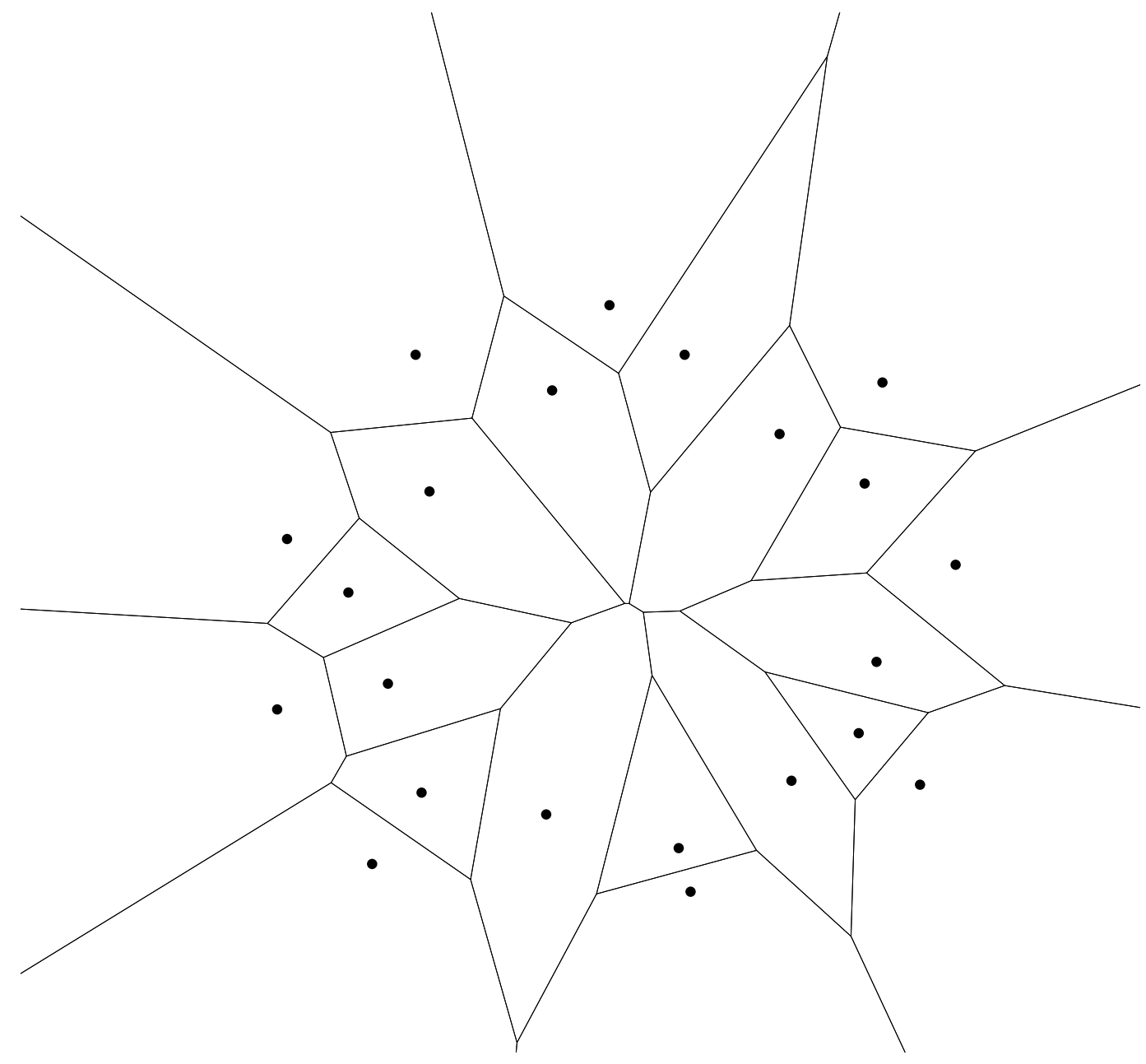

Figure 3: The nearest point Voronoi diagram of a set of points.

$s_{i}$ than from any other element in $S$ (see Figure 4). We denote this region by $V_{F}\left(s_{i}\right)$ and $\operatorname{FPVD}(S)$ is the union of all the regions $V_{F}\left(s_{i}\right), s_{i} \in S$.

- The generalized Voronoi diagram is simply the generalization of the Voronoi diagram defined above, where the input set may now consist of objects other than points. For instance, if the input set $S$ consists of planar points as well as line segments, the Voronoi regions are now bounded by parabolic arcs as well as straight line segments (as opposed to just straight line segments, as is the case for sets of points). In Section 5, we use the generalized nearest point Voronoi diagram of a set consisting of points and circular arcs, where the Voronoi regions are bounded by straight line segments and curves of degree 2 .

An important and useful property of the above diagrams is that the number of 


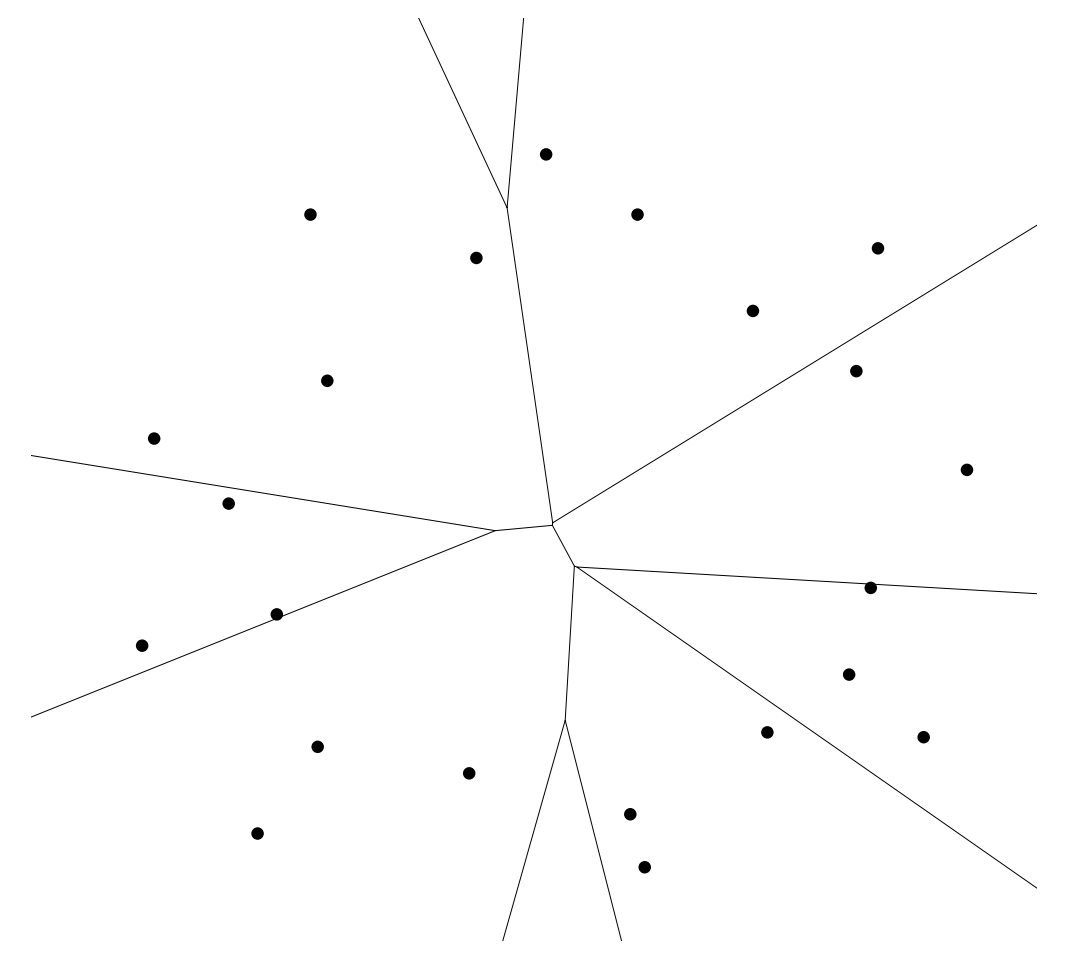

Figure 4: The furthest point Voronoi diagram of a set of points.

Voronoi regions, Voronoi edges and Voronoi vertices are all $O(n)$, where $n$ is the size of the input set.

\section{$3 \quad$ Fixed Outer Radius}

Input $\mathrm{A}$ radius $r$ and a set of points $S$;

Question What is minimum width annulus with outer radius $r$ that contains $S$ ?

Let $F$ be the intersection of the $n$ discs $D\left(s_{i}, r\right), 1 \leq i \leq n$. Any annulus with outer radius $r$ containing every point in $S$ must be centered at some point in $F$. Furthermore, to find the minimum width annulus with outer radius $r$, it is sufficient to find the largest empty circle with center in $F$. In the remainder of this section, we describe a simple $O(n \log n)$ time algorithm to compute the minimum width annulus with outer radius $r$ that contains $S$. Before proceeding, we state the following frequently used fact about closest point Voronoi regions: Given a point $p \in V\left(s_{i}\right)$, the largest empty circle centered at $p$ has radius $d\left(p, s_{i}\right)$.

Lemma 1 Given a planar set of points $S$ and a closed bounded region $R$ contained 


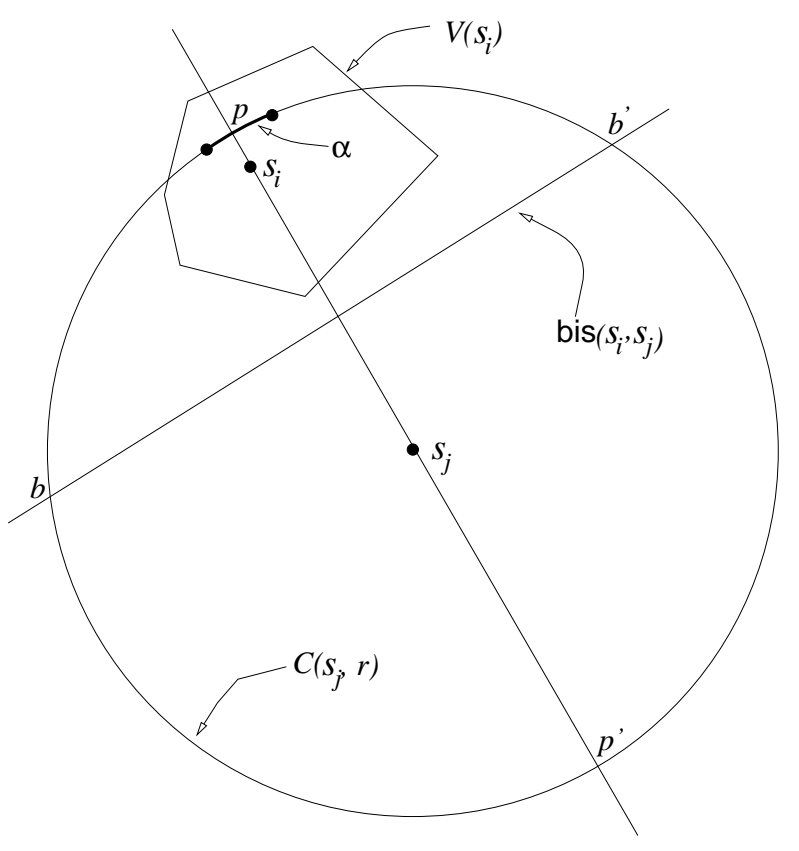

Figure 5: Largest empty circle will be centered at an endpoint of $\alpha$.

entirely in the Voronoi cell of some $s_{i} \in S$, the largest empty circle with center in $R$ is centered at a point on $\mathrm{bd}(R)$.

Proof: Consider a point $p \in \operatorname{int}(R)$. Consider the ray $\overrightarrow{s_{i} p}$ originating at $s_{i}$ and going through $p$. Since $p$ lies in the interior of the closed bounded region $R$, there must be a point $p^{\prime}$ of intersection between $\overrightarrow{s_{i} p}$ and bd $(R)$ such that $d\left(s_{i}, p^{\prime}\right)>d\left(s_{i}, p\right)$. Therefore, given an empty circle centered in the interior of $R$, a larger empty circle can always be found by moving the center to a boundary point. Consequently, since the largest empty circle centered at a point $p$ in $R$ has radius $d\left(s_{i}, p\right)$, the largest empty circle with center in $R$ will be centered at a point on $\operatorname{bd}(R)$ that is furthest from $s_{i}$.

Lemma 2 Let $\alpha \subseteq C\left(s_{j}, r\right)$ be a circular arc contained entirely within a Voronoi region $V\left(s_{i}\right)$. The largest empty circle constrained to have its center on $\alpha$ will have one of the endpoints of $\alpha$ as its center.

Proof: Consider the line going through $s_{j}$ and $s_{i}$. Let $p$ and $p^{\prime}$ be the points of intersection between this line and $C\left(s_{j}, r\right)$ (refer to Figure 5). Assume, without loss of generality, that $d\left(p, s_{i}\right)<d\left(p^{\prime}, s_{i}\right)$. Observe that the distance from $s_{i}$ to a point on $C\left(s_{j}, r\right)$ increases monotonically from $p$ to $p^{\prime}$ (in the counter-clockwise direction, say) and decreases monotonically from $p^{\prime}$ to $p$. 
Let $b$ (respectively, $\left.b^{\prime}\right)$ be the point of intersection between $\operatorname{bis}\left(s_{i}, s_{j}\right)$ and $C\left(s_{j}, r\right)$ encountered while moving from $p$ to $p^{\prime}$ (respectively, $p^{\prime}$ to $p$ ) in the counter-clockwise direction on $C\left(s_{j}, r\right)$. Note that there must be two such points of intersection since the arc $\alpha$ lies in $V\left(s_{i}\right)$ (we may have the degenerate case of $b=b^{\prime}$ in the case that $\alpha$ is just a single point). Clearly, $\operatorname{arc}\left(b, p^{\prime}\right)$ and $\operatorname{arc}\left(p^{\prime}, b^{\prime}\right)$ cannot lie in $V\left(s_{i}\right)$. Hence $\alpha$ must be a subset of the circular arc given by $\operatorname{arc}\left(b^{\prime}, p\right) \cup \operatorname{arc}(p, b)$. From the monotonicity property stated above, it follows that the largest empty circle constrained to have its center on $\alpha$ will be centered at the endpoint of $\alpha$ furthest from $s_{i}$.

Since $F$ is the intersection of a set of discs, it is a convex circle-gon with at most $n$ vertices. Our goal is to compute the intersection of $\operatorname{NPVD}(S)$ and $F$. Note that the number of intersection points on $\operatorname{bd}(F)$ is $O(n)$, since an edge of NPVD $(S)$ intersects bd $(F)$ at most twice.

Let $F\left(s_{i}\right)$ denote the convex region obtained by intersecting $F$ and $V\left(s_{i}\right)$. For each non-empty $F\left(s_{i}\right)$, we find the largest empty circle $\mathcal{C}\left(s_{i}\right)$ with center in $F\left(s_{i}\right)$. From Lemma 1, it follows that $\mathcal{C}\left(s_{i}\right)$ will have its center $c$ on bd $\left(F\left(s_{i}\right)\right)$. Also, $\mathcal{C}\left(s_{i}\right)$ will have radius $d\left(c, s_{i}\right)$. The minimum width annulus with outer radius $r$ will be given by the best of these candidates i.e. by a circle from the set $\left\{\mathcal{C}\left(s_{i}\right) \mid 1 \leq i \leq n\right\}$ that has the maximum radius. Observe that $\mathrm{bd}\left(F\left(s_{i}\right)\right)$ is composed of circular arcs and/or straight line segments (which come from the Voronoi edges of $V\left(s_{i}\right)$ ). We thus have the following characterization of the candidate centers for the minimum width annulus with outer radius $r$.

Theorem 1 The largest empty circle with center in $F$ must be centered at a Voronoi vertex lying in $F$, at a vertex of $\mathrm{bd}(F)$ or at an intersection point between $\mathrm{bd}(F)$ and a Voronoi edge.

Proof: For any straight line segment that is part of bd $\left(F\left(s_{i}\right)\right)$, the largest empty circle must be centered at one of the end points. From this fact and from Lemma 2, it follows that $\mathcal{C}\left(s_{i}\right)$ is given by the largest of the circles centered at the vertices of $\mathbf{b d}\left(F\left(s_{i}\right)\right)$. A vertex of $\mathbf{b d}\left(F\left(s_{i}\right)\right)$ is either a Voronoi vertex, a vertex of $\mathbf{b d}(F)$ or an intersection point between $\mathrm{bd}(F)$ and a Voronoi edge.

We describe below a simple $O(n \log n)$ time algorithm to determine the intersection points between NPVD $(S)$ and bd $(F)$. We would like to point out that it is possible to use Guibas and Seidel's output-sensitive planar-map overlay algorithm [11] to solve our problem within the same time bounds. However, we present here an extremely simple algorithm that is more practical than that implied by the more general algorithm in [11].

Let $\ell(c, \theta)$ be an oriented line, where $c$ is a point on the line and $0 \leq \theta<2 \pi$ is the angle the line makes with the positive $x$-axis. We say that an oriented line 
is a supporting line of a region $R$ if it is incident on $\mathrm{bd}(R)$ and $R$ lies entirely on or to the left of the line. We use $L(R, \theta)$ to denote the supporting line of $R$ that is parallel to $\ell(c, \theta)$. Let $v_{1}, v_{2}, \ldots, v_{n}$ be the vertices of a convex circle-gon $F$ in counter-clockwise order, and let $a_{i}=\left(v_{i}, v_{i+1}\right)$ be its circular-arc edges $\left(v_{n+1} \equiv v_{1}\right)$. The convex polygon $P$ obtained from this vertex set is said to be the polygon defined by $F$. We then have the following.

Lemma 3 Given an oriented line $\ell(c, \theta)$, let $L(P, \theta)$ be incident on vertex $v_{i}$. Then either $L(F, \theta)=L(P, \theta)$, or $L(F, \theta)$ is tangent to one of the arcs $a_{i}$ or $a_{i-1}\left(a_{0} \equiv a_{n}\right)$.

Proof: The oriented line $L(P, \theta)$ either intersects the interior of $F$ or not. It not, then $L(F, \theta)=L(P, \theta)$ since $L(P, \theta)$ is incident on a vertex of $F$. Hence assume that $L(P, \theta)$ intersects the interior of $F$. Since $F$ is convex, $L(P, \theta)$ will intersect $\mathbf{b d}(F)$ at one other point (other than $v_{i}$ ). Call this point $p$. Note that all the vertices of $F$ lie to the left $L(P, \theta)$. (One other vertex of $F$ may lie on $L(P, \theta)$ if it passes through an edge of $P$, in which case $p=v_{i+1}$ or $p=v_{i-1}$.) The point $p$ must lie on one of the two $\operatorname{arcs} a_{i}$ or $a_{i-1}$ because if $p$ were to lie on some other arc, it would imply that $v_{i+1}$ or $v_{i-1}$ lie to the right of $L(P, \theta)$, a contradiction. Assume without loss of generality that $p$ lies on $a_{i}$. Since the line $L(P, \theta)$ intersects the circular arc $a_{i}$, there exists an oriented line tangent to $a_{i}$ and parallel to $L(P, \theta)$. This is the desired supporting line $L(F, \theta)$.

Lemma 4 Given a set of $k$ line segments and a convex circle-gon $F$ with $n$ vertices, whether each segment intersects $F$ and the points of intersection on $\mathrm{bd}(F)$, if any, can be determined in $O(k \log n)$ total time with a simple linear time pre-processing step.

Proof: $\quad$ Let $P$ be the convex polygon defined by $F$. A pair of parallel lines of support of $P$ are two supporting lines $L(P, \theta)$ and $L(P, \theta+\pi)$ such that at least one of them is incident on an edge of $P$. There are at most $n$ combinatorially distinct pairs of parallel lines of support of $P$, which can be found in $O(n)$ time using the well-known technique of rotating calipers [20,24]. We thus obtain a list of supporting lines sorted by angle (with respect to the positive $x$-axis, say).

Let $l$ be a segment from the given set of segments, and $\ell(c, \theta)(0 \leq \theta<\pi)$ and $\ell\left(c, \theta^{\prime}\right)$ (where $\theta^{\prime}=\theta+\pi$ ) be the two oriented lines containing $l$. Let $L(P, \theta)$ (respectively $L\left(P, \theta^{\prime}\right)$ ) be incident on vertex $v_{i}$ (resp. $v_{j}$ ) of $P$; refer to Figure 6. Note that $v_{i}$ and $v_{j}$ can be found in $O(\log n)$ time by using binary search on the sorted list of supporting lines found above. From Lemma $3, L(F, \theta)$ and $L\left(F, \theta^{\prime}\right)$ can then be found in constant time. Let $L(F, \theta)$ (respectively $L\left(F, \theta^{\prime}\right)$ ) be incident at point $p$ (resp. $\left.p^{\prime}\right)$ on $\mathrm{bd}(F)$. 


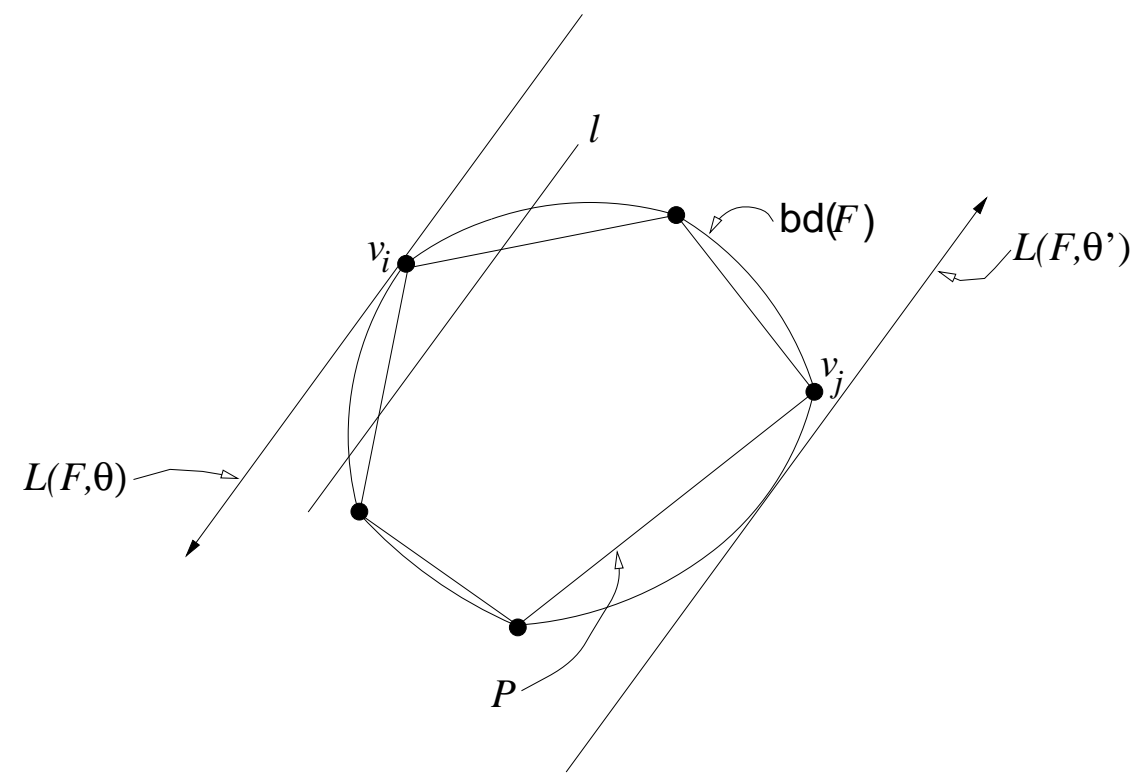

Figure 6: Computing segment intersection with $F$.

- If $\ell(c, \theta)$ lies to the right of $L(F, \theta)$ or $L\left(F, \theta^{\prime}\right)$, then $l$ does not intersect $F$.

- If not, then $\ell(c, \theta)$ must intersect $F$. Observe that one such intersection point must lie on the part of $\operatorname{bd}(F)$ lying between $p$ and $p^{\prime}$, and the other between $p^{\prime}$ and $p$ (in counter-clockwise order). These points of intersection can be found in $O(\log n)$ time using binary search on each of these two lists of circular arcs. We can then determine in constant time if the segment $l$ lies completely inside $F$, completely outside $F$ or intersects bd $(F)$.

By carrying out the above procedure for every Voronoi edge of $\operatorname{NPVD}(S)$, we can identify all Voronoi vertices that lie inside $F$ and also compute all the intersection points between NPVD $(S)$ and bd $(F)$. This takes $O(n \log n)$ time, since there are $O(n)$ Voronoi edges.

For each vertex of $F$, it is necessary to identify the Voronoi region in which it lies. This can be done in $O(\log n)$ time for each vertex, after a linear-time preprocessing step, by using a point location algorithm for planar subdivisions [12]. However, a simple sort step, followed by a linear scan, will give us the desired information within the same time bounds: Sort the vertices and intersection points on bd $(F)$ in counterclockwise order with respect to some point in the interior of $F$ (say the centroid of three vertices of $F$ ). Starting at an intersection point, step through this sorted list. 
1. If we step from an intersection point $p$ to a vertex $v$ of $F$, then let $e \subset \operatorname{bis}\left(s_{i}, s_{j}\right)$ be the Voronoi edge that caused the intersection $p$. If $v$ lies in the half-plane closer to $s_{i}$, then $v$ must lie in $V\left(s_{i}\right)$. If not, it must lie in $V\left(s_{j}\right)$.

2. If we step from a vertex to another vertex, the latter must lie in the same Voronoi region as the former.

Note that if there are no intersection points on bd $(F)$, it means that $F$ lies entirely within some $V\left(s_{i}\right)$, and such an $s_{i}$ can be identified in linear time.

In summary, the outline of the algorithm to compute the minimum width annulus with outer radius $r$ is as follows:

1. Compute the nearest point Voronoi diagram of the set of points $S$ in $O(n \log n)$ time (e.g. $[8,21])$.

2. Compute the intersection $F$ of the $n \operatorname{discs} D\left(s_{i}, r\right), s_{i} \in S$ in $O(n \log n)$ time [6].

3. Identify the Voronoi vertices that lie in $F$ and find the intersection points between $\operatorname{NPVD}(S)$ and $F$ (refer Lemma 4). For each vertex of bd $(F)$, find the Voronoi region in which it lies, as described above. This takes $O(n \log n)$ time.

4. Find the largest empty circle with center in $F$ in $O(n)$ time (refer Theorem 1).

Theorem 2 Given a set $S$ of $n$ points in the plane, the minimum width annulus containing $S$ and having outer radius $r$ can be computed in $O(n \log n)$ time.

\section{$4 \quad$ Fixed Inner Radius}

Input: A radius $r$ and a set of points $S$;

Question: What is minimum width annulus with inside radius $r$ that contains $S$ ?

Let $U$ be the union of open discs $\widetilde{D}\left(s_{i}, r\right), 1 \leq i \leq n$. Note that the center of an annulus with fixed inner radius $r$ must be outside the union of these open discs since by construction there is no empty circle of radius $r$ whose center lies inside the union. Therefore, the center of the minimum spanning disc of $S$ whose center lies outside $U$ is the center of the thinnest annulus with fixed inner radius $r$. In the following two lemmas, we first determine the different configurations of points of $S$ that must be on the boundary of the thinnest annulus and then use these configurations to show that there exists a linear size set $C$ of locations that contains the center of the thinnest annulus with fixed inner radius $r$. This implies a natural approach to computing 


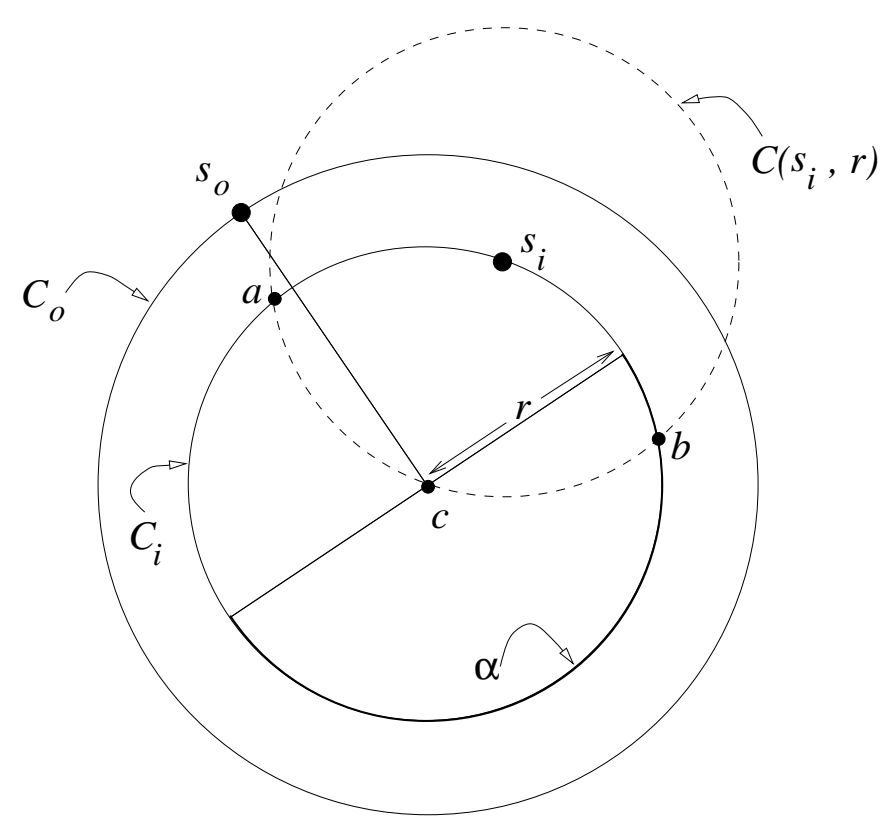

Figure 7: Illustration for Lemma 5.

the thinnest annulus. First, compute the set $C$ of candidate locations for the center. Second, for each location in $C$, compute the thinnest annulus. Output the smallest of these annuli. The fact that the true center must be in $C$ guarantees that the output is the thinnest annulus.

Let $F$ be the complement of $U$ (i.e. $F$ is the complement of the union of $n$ open discs, each of radius $r$ centered at each $s \in S$ ). The centroid of a pair of points is defined as the midpoint of the line segment between them.

Call an annulus feasible if it contains all of the points in $S$.

Lemma 5 The minimum width feasible annulus with fixed inner radius $r$ must have (at least)

1. three points or a diametral pair on the outer circle,

2. two points on the inner circle and one on the outer, or

3. two points on the outer and one point on the inner.

Proof: We argue that if case 1 does not hold, then one of the other cases must hold. Suppose that case 1 does not hold. If the outer circle contains no points, we can find a thinner feasible annulus by shrinking the outer circle. If the outer circle contains a single point $s$ (or a non-diametral pair $\left(s_{1}, s_{2}\right)$ ), and the inner circle contains no points, we can find a thinner feasible annulus by translating the center towards $s$ (or 
along bis $\left.\left(s_{1}, s_{2}\right)\right)$ until either the outer or the inner circle hits a new point. Note that since the radius of the outer circle is shrinking and that of the inner circle is fixed, this new annulus is thinner. Now suppose both the inner circle $C_{i}$ and the outer circle $C_{o}$ each contain exactly one point, $s_{i}$ and $s_{o}$ respectively. Let $c$ denote the center. Consider the diameter of $C_{i}$ that is perpendicular to the segment $\overline{c s_{o}}$ and let $\alpha$ be the open semicircle of $C_{i}$ that $\overline{c s_{o}}$ does not intersect (see Figure 7). If $s_{i} \in \alpha$, we can find a thinner feasible annulus by translating $c$ towards $s_{o}$ (this is similar to the case described earlier). Otherwise, consider the circle $C\left(s_{i}, r\right)$. Let $a$ and $b$ be the two intersection points between $C\left(s_{i}, r\right)$ and $C(c, r)$. Since $s_{i} \notin \alpha$, there must be an intersection point, say $a$, not in $\alpha$. Consider the arc (of $C\left(s_{i}, r\right)$ ) from $c$ to $a$ not containing $b$. As we move a point $x$ along this arc, the distance $d\left(x, s_{o}\right)$ gets strictly smaller, which implies that we can find a thinner feasible annulus.

Lemma 6 There are $O(n)$ positions where the the center of the thinnest annulus for $S$ constrained to have center in $F$ can be located. They are the following:

1. the midpoint of the diameter of $S$,

2. a vertex of $\operatorname{FPVD}(S)$,

3. the intersection point of an edge e of $\operatorname{FPVD}(S)$ and $\mathrm{bd}(F)$ closest to the centroid of the the two generators of $e$,

4. a vertex $v$ of $\mathrm{bd}(F)$.

Proof: We use the configurations outlined in Lemma 5 to prove that the center can be located only in one of the four places enumerated in the statement of the lemma which we shall refer to as $\mathrm{P} 1, \mathrm{P} 2, \mathrm{P} 3$, and $\mathrm{P} 4$, respectively. Let $A$ be the thinnest annulus constrained to have center in $F$ with outer circle $O$ and inner circle $I$. Let the radius of $O$ be $r_{O}$ and the center of $O$ be $c_{O}$. The radius of $I$ is fixed to be $r$ and the center is also $c_{O}$. We address each of the possible configurations of points that can lie on the boundary of $A$.

\section{Three points or a diametral pair on the outer circle.}

If there are three points on the outer circle, then the center must be a vertex of the $\operatorname{FPVD}(S)$, therefore it is located in P2. If there is a diametral pair of points on the outer circle, then that pair of points is a diameter of the set $S$. Therefore the center lies in P1.

\section{Two points on the inner circle and one on the outer.}

Let the two points on the inner circle be $s_{i}$ and $s_{j}$. The center must lie on the boundary of the two circles of radius $r$ centered around these two points, $C\left(s_{i}, r\right)$ 
and $C\left(s_{j}, r\right)$ respectively. Therefore, it must be on a vertex of the boundary of $F$ which is $\mathrm{P} 4$.

\section{Two points on the outer and one point on the inner.}

If there are two points $s_{i}$ and $s_{j}$ on the outer circle then the center lies on an edge $e$ of the FPVD $(S)$ since it is equidistant from two points on the boundary of a spanning disc. There is one point $s_{k}$ on the inner circle. Therefore, the center must also lie on the boundary of $F$. Thus, the center lies on the intersection of an edge $e$ with the boundary of $F$. We need to show that this point is the closest point to the centroid of the two generators $s_{i}$ and $s_{j}$ of the edge $e$. Suppose that it is not the closest. Let $c$ be the intersection point of $e$ and $\mathrm{bd}(F)$ closest to the centroid. We note that $c$ is still a center for a spanning disc and that $d\left(c, s_{i}\right)$ is strictly less that $d\left(c_{O}, s_{i}\right)$ contradicting the fact that $A$ is minimum. Therefore, the center lies in P3.

There are $O(n)$ midpoints of the diameter of $S[16,17]$. There are $O(n)$ vertices in $\operatorname{FPVD}(S)[16,17]$. There are $O(n)$ edges in $\operatorname{FPVD}(S)[16,17]$, which implies that there are only $O(n)$ intersection points of an edge $e$ of $\operatorname{FPVD}(S)$ and $\operatorname{bd}(F)$ closest to the centroid of the two generators of $e$. Finally, $F$ has $O(n)$ vertices [6]. Therefore, there are $O(n)$ candidate locations for the center of the thinnest annulus of $S$ constrained to having a center in $F$.

The approach used to solve the problem is to verify each of the candidate positions for the center as shown in Lemma 6 and select the position yielding the smallest spanning disc. The complement of the union of $n$ open discs has linear boundary complexity [6]. The edges of the boundary are arcs of circles of radius $r$ and the vertices are the intersections of two or more arcs. Given a set of $n$ open discs, the complement of their union can be computed in $O(n \log n)$ time [4,6]. Therefore, the set $F$ can be computed in $O(n \log n)$ time.

As Lemma 6 points out the four types of locations where the center can lie, we show how to enumerate these candidate positions and then show how to test each one in order to find the center of the minimum spanning disc. The total time taken to enumerate and test the candidate centers is $O(n \log n)$.

First, the centers of the diameter $d_{i}$ of $S$ can be computed in $O(n \log n)$ time [17]. Second, we compute all of the vertices of the $\operatorname{FPVD}(S)$. We need determine if a center of a diameter of $S$ or a vertex of $\operatorname{FVD}(S)$ is in $F$. To do this, we first compute in $O(n \log n)$ time the closest point Voronoi diagram of $S$, denote $\operatorname{NPVD}(S)[8,16,17$. We preprocess NPVD $(S)$ for point location [12] (i.e. with $O(n)$ preprocessing, we can locate the cell of $\operatorname{NPVD}(S)$ containing a query point in $O(\log n)$ time). For each 
center $d_{i}$ of the diameter of $S$ and each vertex $v_{i}$ of $\operatorname{FPVD}(S)$, we determine which cell of NPVD $(S)$ it lies in. If the distance between $d_{i}$ (resp. $v_{i}$ ) and the generator of the cell it is in is at least $r$, then $d_{i}$ (resp. $v_{i}$ ) lies inside $F$. Therefore, since there are $O(n)$ centers of the diameter of $S$ and $O(n)$ vertices in $\operatorname{FPVD}(S)$, generating all these candidate centers can be accomplished in $O(n \log n)$ time.

Third, we need to compute every intersection point of an edge $e$ of $\operatorname{FPD}(S)$ and $\operatorname{bd}(F)$ closest to the centroid of the two generators of $e$. Let $p$ and $q$ be the two points that generate edge $e$. Let $p^{*}$ be the intersection of the line containing $e$ and segment $\overline{p q}$. We first test if $p^{*}$ is in $e$ and in $F$. If it is, then it is a candidate center and we generate it. Since there are only a linear number of edges in $\operatorname{FPVD}(S)$ there can only be a linear number of such candidate centers. If $p^{*}$ is not in $e$ and $F$, then conceptually we perform two ray shooting queries along the line containing $e$ in the two directions away from $p^{*}$ to find the two closest feasible centers to $p^{*}$. If these intersection points are on $e$, then we generate them. Once again, for each $p^{*}$ we generate at most two intersection points, therefore there are at most $O(n)$ such candidate centers.

Preprocessing $F$ for ray shooting queries is too costly, so to generate the latter set of candidates, we perform two plane sweeps. The boundary of $F$ is broken into $y$-monotone pieces and we perform one sweep for left rays away from $p^{*}$ and one for right rays. After the first intersection for a given ray is found, we remove it from the event queue. Each ray is therefore processed at most twice, once when it is placed in the queue and once for its first intersection. Since there are only $O(n)$ rays and the boundary of $F$ has $O(n)$ size, each of the two sweeps takes $O(n \log n)$ time to generate the $O(n)$ candidate centers. For more details on how to implement a plane sweep, refer to O'Rourke [16] or Preparata and Shamos [17].

Finally, each vertex of $F$ is a candidate center. Thus, there are only $O(n)$ such candidate centers and they can be generated in $O(n \log n)$ time $[4,6]$.

Once all of the $O(n)$ candidates have been generated, computing the location of the center and the radius of the minimum spanning disc is simple. For each candidate center $c_{i}$, we determine in which cell of the $\operatorname{FPVD}(S)$ it lies. This can be accomplished in $O(\log n)$ time per candidate with $O(n)$ preprocessing time [12]. The distance between $c_{i}$ and the generator of the cell containing $c_{i}$ is the length of the radius of the spanning disc. We output the smallest spanning disc. Since none of the steps outlined above take more than $O(n \log n)$ time, we conclude with the following theorem.

Theorem 3 Computing the thinnest annulus with inner radius fixed at $r$ of $n$ points in the plane can be accomplished in $O(n \log n)$ time. 


\section{$5 \quad$ Fixed Median Radius}

Input A radius $r$ and a set of points $S$;

Question What is minimum width annulus with median radius $r$ that contains $S$ ?

Call an annulus feasible if it contains every element of the input set $S$.

Lemma 7 The minimum width feasible annulus of given median radius $r$ contains either a diametral pair on the outer circle or at least 3 elements of $S$ on its boundary.

Proof: We argue that if some feasible annulus $A$ contains less than three points of $S$ on its boundary, but not a diametral pair on the outer circle, we can perturb $A$ to another feasible annulus of the same median radius, but with strictly smaller width.

If the boundary of $A$ contains 0 points of $S$, we can decrease the outer radius while increasing the inner radius until one of the bounding circles encounters a point of $S$.

If the boundary of $A$ contains 1 point $p$ of $S$, draw the line $L$ through the center of $A$ and $p$. If $p$ is on the inner (outer) circle translate the center of the annulus away from (towards) $p$ along $L$ while keeping the inner (outer) circle touching $p$; at the same time shrink or grow the other circle at the appropriate rate to maintain the median radius.

The case of two non-diametral contact point on the same circle of the boundary is similar to that of one, except that the center of the annulus is translated along the bisector of the two points. If we have a diametral pair on the inner circle then we have two choice for a direction to translate the center.

Consider the case of one contact point $o$ on the outer circle and one point $i$ on the inner. We want to translate the center $c$ of the annulus to some new center $c^{\prime}$ for the annulus that maintains the invariant $d\left(i, c^{\prime}\right)+d\left(o, c^{\prime}\right)=2 r$. This defines an ellipse of possible centers; the center $c$ must be in the open half ellipse that maintains the invariant $d(i, c)<d(o, c)$. It follows that in the neighborhood of $c$ there must be some direction along the ellipse that strictly decreases the distance to $o$ and strictly increases the distance to $i$, thus strictly decreasing the width of the annulus.

Place a disc of radius $2 r$ centered at each point in $S$; this is the maximum possible outside radius, since it drives the inside radius to zero. In the rest of this section let $F$ denote the intersection of these discs. Any feasible annulus with median radius $r$ must have a center in $F$; conversely for every point in $F$, there is a feasible annulus of median radius $r$ centered at that point. The region $F$ is a circle-gon, a convex region bounded 
by circular arcs. For a point $p$ and a set $X$, let $d_{\min }(p, X)$ denote $\min _{x \in X} d(p, x)$. We say that $C(s, 2 r)$ is a closest circle to $p$, if for all $s^{\prime} \in S d_{\min }\left(p, C\left(s^{\prime}, 2 r\right)\right) \geq$ $d_{\min }(p, C(s, 2 r))$. For any point $p$ in $F, d_{\min }(p, C(s, 2 r))=2 r-d(p, s)$. Thus we have the following:

Lemma 8 For any $p \in F$, if $s$ is a farthest element of $S$ from $p$, then $C(s, 2 r)$ is a closest circle to $p$.

Rather than concerning ourselves with the intersection of two Voronoi diagrams as in the characterization of candidate centers given in Section 1, the previous lemma allows us to compute the generalized (nearest point) Voronoi diagram of the points $s$ and certain arcs of the circles $C(s, 2 r)$. In general we could break each circle $C(s, 2 r)$ into some constant number circular arcs and use all of these arcs as sites in the Voronoi diagram. In order to ensure that the arcs intersect only at their endpoints (which is necessary to ensure a linear bound on the size of the generalized Voronoi diagram), we show that we need only concern ourselves with the arcs bounding $F$.

Lemma 9 For any $p \in F$, if $C(s, 2 r)$ is a closest circle to $p$, then an arc of $C(s, 2 r)$ containing the point on $C(s, 2 r)$ closest to $p$ is part of the boundary of $F$.

Proof: $\quad$ Let $q$ be the point on $C(s, 2 r)$ nearest $p$. Suppose some other circle $C\left(s^{\prime}, 2 r\right)$ intersects line segment $\overline{p q}$. Then $d_{\min }\left(p, C\left(s^{\prime}, 2 r\right)\right)<d_{\min }(p, C(s, 2 r))$, a contradiction.

Lemma 7 characterized candidate centers for the minimum width annulus of median radius $r$ as being either midpoints of diameters of the input set $S$, or as those points $c$ such that the minimum width feasible annulus of median radius $r$ contains at least three points of $S$ on its boundary. Notice that the second type of candidate are exactly the vertices of the generalized Voronoi diagram $G$ of $S$ and the boundary of $F$. Each vertex of $G$ is closest to three sites; in the case where a site is some $s \in S$, this corresponds to $s$ being on the inner boundary of the annulus with median radius $r$ and centered at the vertex. In the case where a closest site is an arc of $C(s, 2 r)$ (part of the boundary of $F$ ), by Lemma 8 and Lemma 9 this corresponds to $s$ being on the outer boundary of the annulus with median radius $r$. We summarize in a theorem.

Theorem 4 Let $G$ be the generalized Voronoi diagram of $S$ and the arcs bounding $F$. The center of the minimum enclosing annulus of median radius $r$ is either a vertex of $G$ contained in $F$ or the center $c_{d} \in F$ of a diameter of $S$.

The outline of our algorithm is as follows.

1. Compute $F$ in $O(n \log n)$ time by divide and conquer [6]. 
2. As in the previous section, we can compute the centers of the diameter of $S$ in $O(n \log n)$ time.

3. Compute $G$ in $O(n \log n)$ time by the algorithm of Yap [25].

4. Test each vertex of $G$, and each diameter center, as follows.

(a) $O(\log n)$ time for point location to determine feasibility.

(b) $O(1)$ time to compute the width of the candidate annulus.

Testing a candidate vertex $v$ of $G$ involves first determining if $v \in F$ and if so computing the minimum width median radius $r$ annulus centered at $v$. Testing a point $p$ for inclusion in $F$ proceeds much as if $F$ were a convex polygon. Choose some arbitrary point $c$ interior to $F$ (say the centroid of the vertices of $F$ ). Draw rays from $c$ through the vertices of $F$, dividing the plane into sectors. Determine by binary search in $O(\log n)$ time which sector $p$ lies in. Each sector corresponds to a unique $\operatorname{arc} A$ of the boundary of $F$, which in turn lies on a unique circle $C(s, 2 r)$. The point $p$ is inside $F$ if and only if it is inside $C(s, 2 r)$.

To compute the width of a minimum width annulus of median radius $r$ centered at a vertex $v$ of $G$, we need only consider a single generator $g$ (arc or point) of $v$. If $g$ is an element of $S$ then the radius of the inner circle is $d(v, g)$. If $g$ is an arc of some circle $C(s, 2 r)$, then the radius of the outer circle is $d(v, s)=2 r-d(v, g)$. In either case, once we have computed one radius $r_{g}$, the radius of the other circle is given by $2 r-r_{g}$. If our candidate center is a center of the diameter of $S$, then the outer radius is naturally half of the diameter; the inner radius is determined as before.

Theorem 5 Given a set $S$ of $n$ points in the plane, the minimum width annulus containing $S$ and having median radius $r$ can be computed in $O(n \log n)$ time.

\section{Conclusions}

We have presented efficient algorithms to compute the thinnest annulus containing a set of $n$ points whose outer (or inner, or median) radius has a given value $r$. This is useful for testing whether a manufactured disc of radius $r$ is within tolerance. Our algorithms run in $O(n \log n)$ time. Hence, they are more efficient than the best known algorithm for computing the thinnest annulus containing a set of points whose radius is not given, which runs in $O\left(n^{3 / 2+\epsilon}\right)$ time.

Acknowledgments: We would like to thank Godfried Toussaint for organizing and inviting us to the workshop on Computational Metrology, held at the Bellairs Research Institute in Barbados in February 1995. We would also like to thank all the participants of the workshop for interesting and useful discussions. 


\section{References}

[1] P.K. Agarwal, B. Aronov, and M. Sharir. Computing envelopes in four dimensions with applications. In Proceedings of the 10th Annual ACM Symposium on Computational Geometry, pages 348-358, 1994.

[2] P.K. Agarwal and M. Sharir. Efficient randomized algorithms for some geometric optimization problems. In Proceedings of the 11th Annual ACM Symposium on Computational Geometry, pages 326-335, 1995.

[3] P.K. Agarwal, M. Sharir, and S. Toledo. Applications of parametric searching in geometric optimization. Journal of Algorithms, 17:292-318, 1994.

[4] F. Aurenhammer. Improved algorithms for discs and balls using power diagrams. Journal of Algorithms, 9:151-161, 1988.

[5] P. Bose and L. Devroye. Intersections with random geometric objects. Technical report, McGill University, 1996.

[6] K. Q. Brown. Geometric transforms for fast geometric algorithms. PhD thesis, Carnegie-Mellon University, Pittsburgh, PA, 1980.

[7] H. Ebara, N. Fukuyama, H. Nakano, and Y. Nakanishi. Roundness algorithms using the Voronoi diagrams. In Proceedings of the First Canadian Conference on Computational Geometry, page 41, 1989.

[8] S. J. Fortune. A sweepline algorithm for Voronoi diagrams. Algorithmica, 2:153$174,1987$.

[9] L. W. Foster. GEO-METRICS II: The application of geometric tolerancing techniques. Addison-Wesley Publishing Co., 1982.

[10] J. García and P. A. Ramos. Circularity of a set of points. Technical Report 4, Departamento de Matematica Aplicada, E.U. Informatica, Universidad Politécnica de Madrid, 1996.

[11] L. J. Guibas and R. Seidel. Computing convolutions by reciprocal search. Discrete Comput. Geom., 2:175-193, 1987.

[12] D. G. Kirkpatrick. Optimal search in planar subdivisions. SIAM J. Computing, $12: 28-35,1983$.

[13] K. Lai and J. Wang. A computational geometry approach to geometric tolerancing. In 16th North American Manufacturing Research Conference, pages 376-379, 1988. 
[14] V.B. Le and D.T. Lee. Out-of-roundness problem revisited. In IEEE Trans. Pattern Anal. Mach. Intell., volume PAMI-13, pages 217-223, 1991.

[15] N. Megiddo. Applying parallel computation algorithms in the design of serial algorithms. Journal of the ACM, 30:852-865, 1983.

[16] J. O'Rourke. Computational Geometry in C. Cambridge University Press, 1994.

[17] F. Preparata and M. I. Shamos. Computational Geometry. Springer-Verlag, 1985.

[18] T. J. Rivlin. Approximation by circles. Computing, 21:93-104, 1979.

[19] U. Roy and X. Zhang. Establishment of a pair of concentric circles with the minimum radial separation for assessing roundness errors. Computer Aided Design, 24(3):161-168, 1992.

[20] M. I. Shamos. Computational Geometry. PhD thesis, Yale University, 1978.

[21] M.I. Shamos and D. Hoey. Closest-point problems. In Proc. Sixteenth Annual IEEE Symposium on Foundations of Computer Science, pages 151-162, October 1975 .

[22] M. Smid and R. Janardan. On the width and roundness of a set of points in the plane. In Proceedings of the Seventh Canadian Conference on Computational Geometry, pages 193-198, Quebec City, Quebec, August 1995.

[23] K. Swanson. An optimal algorithm for roundness determination on convex polygons. In Proc. 3rd Workshop on Alg. and Data Structures, volume 709 of LNCS, pages 601-609, Berlin, 1993. Springer-Verlag.

[24] G. Toussaint. Solving geometric problems with the "rotating calipers". In Proceedsings of IEEE MELECON, pages A10.02/1-4, Athens, Greece, 1983.

[25] C.K. Yap. An $O(n \log n)$ algorithm for the Voronoi diagram of a set of simple curve segments. Discrete Comput. Geom., 2:365-393, 1987. 